\title{
Mini Implant Assisted Correction of a Skeletal Class II Malocclusion with Deep Overbite - A Case Report
}

\author{
Sarah A*, Sandhya M, Tariq M and Saba K \\ Department of Orthodontics and Dentofacial Orthopedics, Aligarh Muslim University, \\ India
}

*Corresponding author: Sarah Asif, Department of Orthodontics and Dentofacial

\section{Case Report \\ Volume 3 Issue 4}

Received Date: October 02, 2018

Published Date: October 15, 2018

DOI: 10.23880 /oajds-16000199 Orthopedics, Ziauddin Ahmad Dental College, Aligarh Muslim University, India, Email: sarahasif87@gmail.com

\section{Abstract}

Case report of a 19 year old male patient who presented with a skeletal Class II malocclusion associated with $100 \%$ deep overbite, vertical maxillary excess, incompetent lips and a hyperdivergent growth pattern. Fixed orthodontic mechanotherapy using preadjusted edgewise appliance along with segmented arch mechanics and mini implants, was used for the correction of the malocclusion and to achieve an optimum soft tissue balance. The treatment outcomes achieved were; correction of overjet and overbite, correction of class canine and molar relation and competency of lips resulting in improvement of facial esthetics.

Keywords: Skeletal Class II malocclusion; Incompetent Lips; Intrusion; Mini Implants; Vertical Maxillary Excess

\section{Introduction}

Deep bite is said to be one of the most challenging malocclusions for an orthodontist to treat and correct. A variety of treatment modalities and biomechanics have been outlined and employed by various researchers till to date, that aid in achieving deep bite correction. Nonsurgical treatment modalities for correction of deep bite are usually directed towards relative mechanics; either by extrusion of posterior teeth or by intrusion of incisors or a combination of both [1-6].

Davidovitch \& Rebellato delineated a variety of factors such as smile line, incisor display, and vertical dimension depending on which the choice of the most suitable treatment option for an individual is opted for [7]. The most routinely employed techniques for deep bite correction are: Cetlin's appliance, RCS wires, Rickett's utility arch, Burstone's intusion spring and arch, KSIR loop, Connecticut intrusion arch, PG retraction spring by Gjessing and the anterior bite plate $[2,8]$.

Recently, mini-screw implants are increasingly being used by orthodontists as the most favorable treatment option for intrusion of teeth and correction of deep bite. Owing to their small dimensions, they provide the benefit of immediate loading, multiple placement sites, relatively simple placement and removal, placement in interdental areas where traditional implants cannot be placed, and minimal costs for patients as well [6].

\section{Case Report}

A 19 year old male reported to the department of orthodontics with the chief complaint of forwardly placed upper front teeth and a non pleasing smile. On extra oral 
examination the patient had an apparently symmetrical mesoprosopic face with a convex profile, incompetent lips and a deep mentolabial sulcus. Smile analysis showed a high smile line with a non-consonant smile arc and a morley's ratio of $100 \%$. On intraoral examination the patient had a class II molar relationship on right side, class I relationship on left side and class II canine relationship bilaterally. Further examination revealed a $100 \%$ deep bite with an overjet of $6 \mathrm{~mm}$, U- shaped maxillary arch and an asymmetric ovoid mandibular arch with crowding in lower anterior region (Figures $1 \& 2$ ).
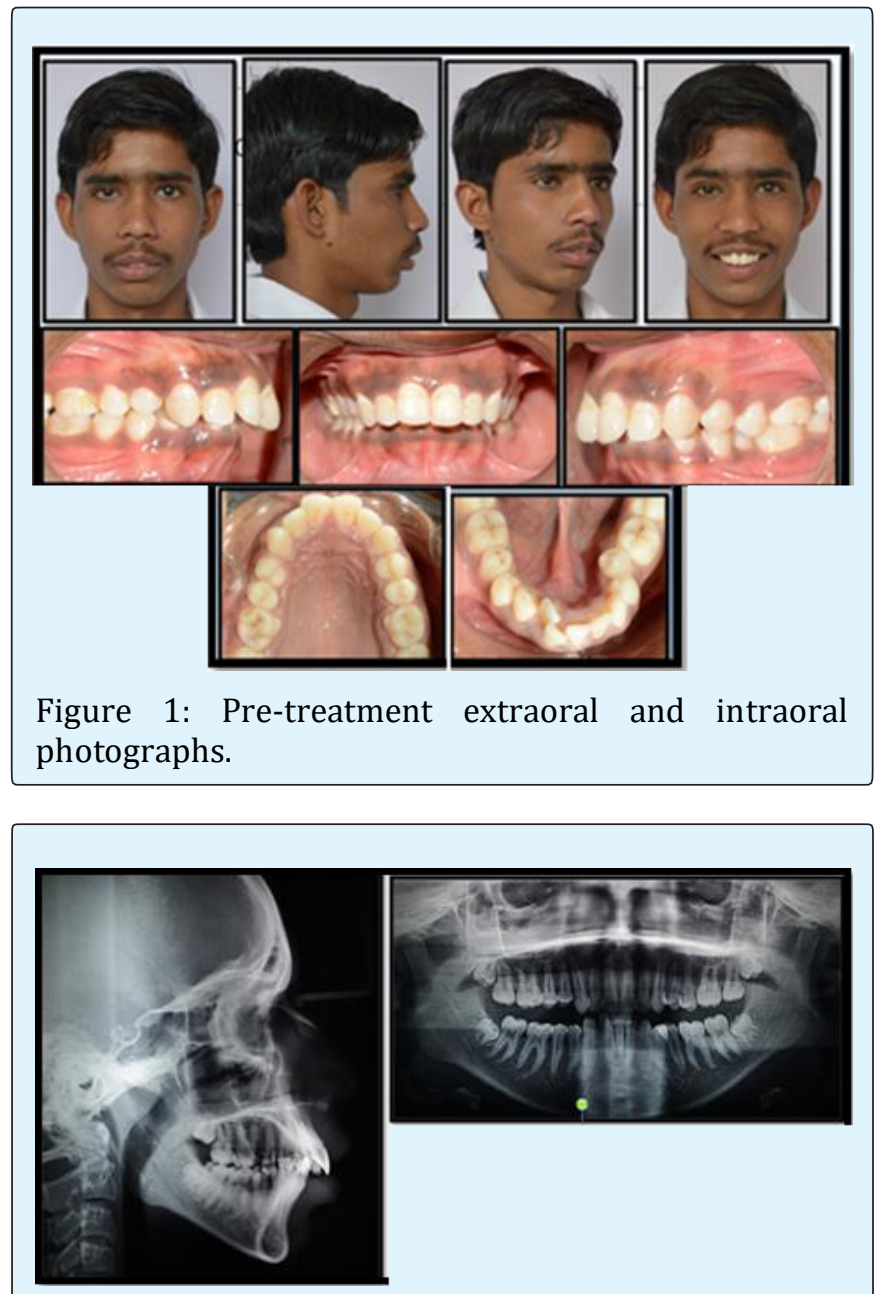

Figure 2: Pre-treatment Extraoral radiographs.

Cephalometric findings presented a forwardly placed maxilla with a normally positioned mandible resulting in a Skeletal class II relation $(\mathrm{ANB}=7$ \& Wits $=6 \mathrm{~mm})$. The cephalometric dentoalveolar findings revealed an increased upper anterior dental height $(\mathrm{Mx} 1-\mathrm{NF}=32 \mathrm{~mm})$ and normal upper and lower posterior dental heights (Mx6-NF=21.5mm and Mand 6- Go-Me=30mm). The patient had a hyperdivergent growth pattern (FMA $=32^{\circ}$, Gonial angle $=135^{\circ}$ and $\mathrm{SN}-\mathrm{MP}=38^{\circ}$ ) .The periodontal tissues were found to be healthy. The functional findings showed no signs and symptoms of a temporomandibular disorder.

Soft tissue cephalometric analysis revealed protrusive upper and lower lip with respect to E line, an increased interlabial gap of $4 \mathrm{~mm}$ and a lip strain of $3 \mathrm{~mm}$. The panoramic X-ray revealed the presence of upper and lower third molars with no apparent pathologies.

\section{Diagnosis and Treatment Objectives}

The patient was diagnosed having an Angle's Class II subdivision malocclusion associated with a complete deep bite, a vertical maxillary excess, gummy smile and incompetent lips on a Skeletal Class II base with a hyperdivergent growth pattern.

The treatment goals were:

a) Correction of Incisor protrusion, Deep bite and Overjet

b) Relieve lower anterior crowding

c) Achieving bilateral Class I Molar and Canine relationship bilaterally

d) Reduce the display of the Maxillary incisors

e) Achieving lip competency and enhancing soft tissue balance.

f) Achieving stable occlusal function

\section{Treatment Plan}

Considering the cephalometric hard tissue and soft findings and model analysis a treatment plan was outlined to extract the maxillary first premolars and mandibular second premolars so as to achieve correction of the vertical as well as the anterio-posterior discrepancy.

\section{Treatment Sequence}

The maxillary and mandibular arches were banded and bonded using preadjusted edgewise appliance (MBT $0.022 \times 0.028$ slot) and lower second premolars were extracted simultaneously to aid in decrowding of the lower arch. An initial 0.016" NiTi wire was placed in both the arches for levelling and alignment. At the end of four months, optimum levelling and aligning was achieved to place upper and lower $0.019 \times 0.025$-inch SS wires. Two mini implants (dimensions $1.5 \mathrm{~mm} \times 8 \mathrm{~mm}$ ) were placed in the upper arch between the canines and the lateral incisor region and two mini implants (dimensions $1.5 \mathrm{~mm} \times 9$ $\mathrm{mm}$ ) were positioned in the lower arch between first and 
second molar region. The positions of each implant was verified on intra oral periapical radiographs.

After successful placement of implants was achieved, the loading of implants was initiated. In the upper arch the mini implants were used for intrusion, by directly attaching NiTi closed coil springs from the wire segment to the implant on each side. Light intrusive forces in the range of 50-60gm were delivered. In the lower arch implant supported retraction of the segment anterior to the extraction sites was initiated. The residual lower extraction space, in the later stages of space closure phase, was utilized for mesializaion of molars. Simultaneously in the upper arch, segmental arch mechanics was used for retraction of the canine (Figure 3 ). After a period of 6 months, optimum retraction of the upper canines and of the lower segment was achieved along with sufficient amount of intrusion of $4.5 \mathrm{~mm}$ in the upper anterior segment.

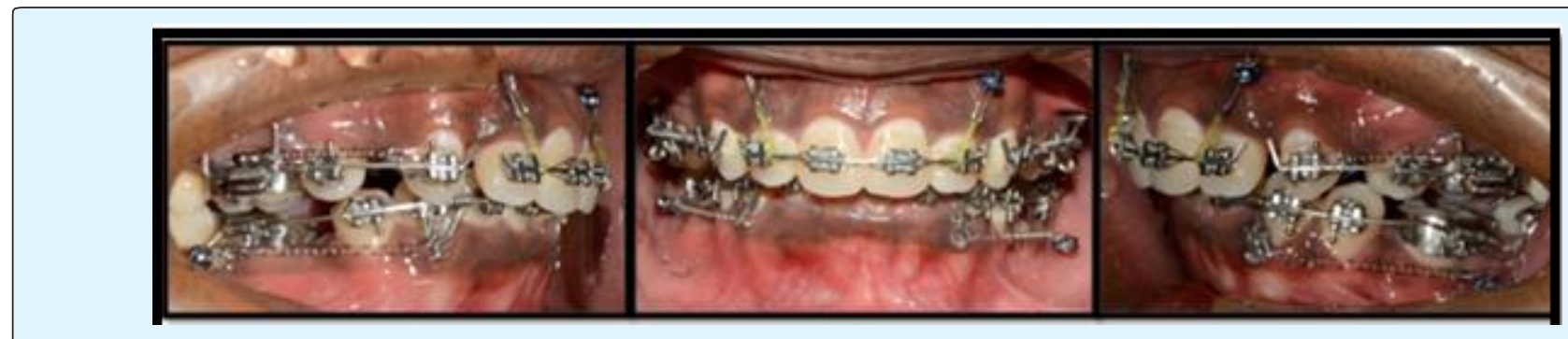

Figure 3: Mini implant assisted intrusion in upper anterior segment and retraction in the lower segment.

For the settling of occlusion, 0.016 nickel titanium wire was placed with settling elastics. Following it, the case was debonded and a fixed upper and lower lingual bonded retainers were given. The total duration of the treatment was 2 years and 7 months. The patient was then advised for timely follow up.

\section{Post Treatment Results}

Post treatment extraoral photographs revealed a marked improvement in the facial profile and esthetics of the patient. Lip competency was achieved and lip strain was relieved. The radiographic evaluation confirmed the good control of the upper and lower labial segments. A significant amount of upper incisor intrusion $3 \mathrm{~mm}$ was attained. An initial bite opening of $4.5 \mathrm{~mm}$ was achieved; however following debonding $2 \mathrm{~mm}$ of relapse was seen , beyond that there was no relapse seen further on routine follow ups and the correction achieved was stable. The hyper divergence of the patient was also controlled well. The lip strain and upper and lower lip protrusion were reduced significantly thereby resulting in labial competency Figures 4-6.

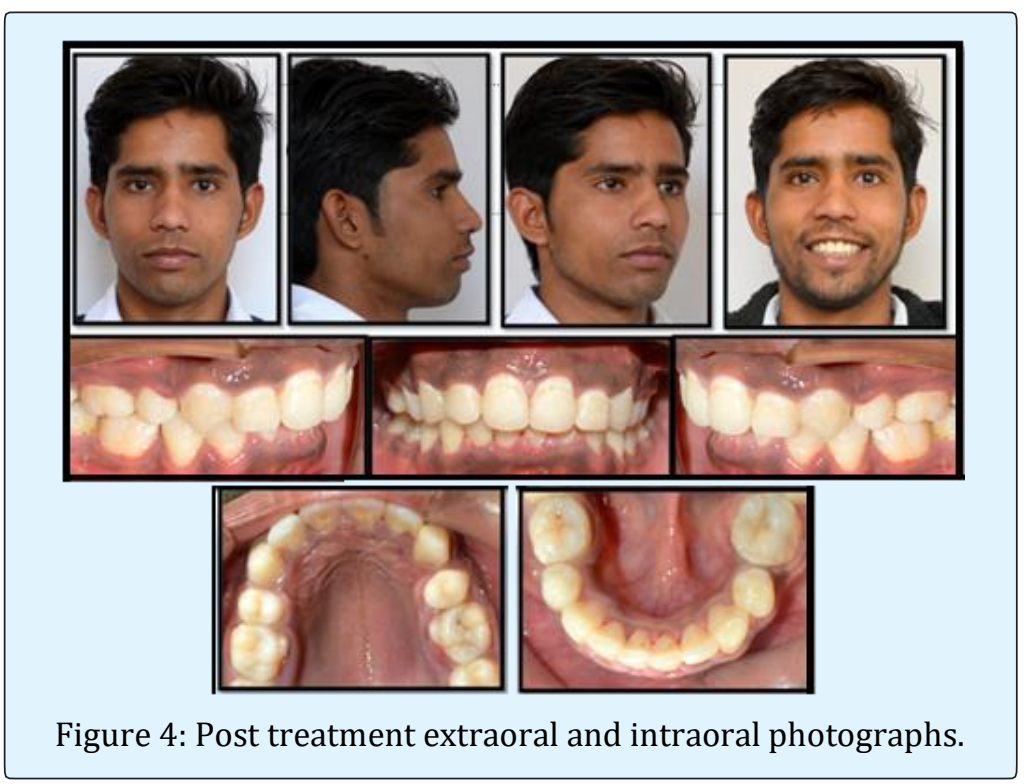

Sarah A, et al. Mini Implant Assisted Correction of a Skeletal Class II Malocclusion with Deep Overbite - A Case Report. J Dental Sci 2018, 


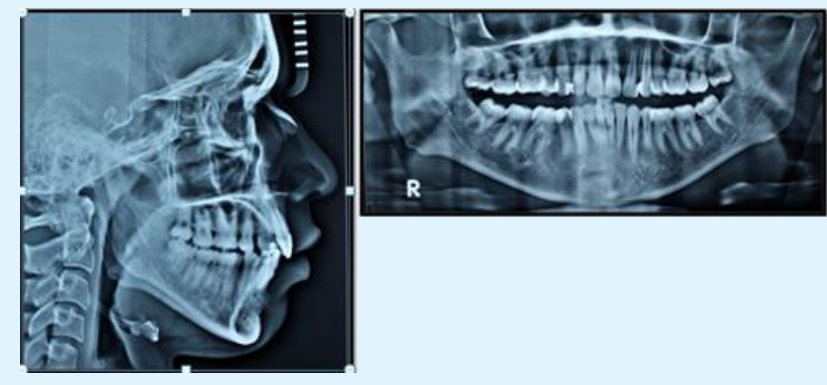

Figure 5: Post treatment extraoral radiographs.

\begin{tabular}{|c|c|c|}
\hline & & \\
\hline Cephalometric parameters & Pre-treatment & Post treatment \\
\hline SNA & $85^{\circ}$ & $84^{\circ}$ \\
\hline SNB & $76^{\circ}$ & $77^{\circ}$ \\
\hline ANB & $9^{\circ}$ & $7^{\circ}$ \\
\hline FMA & $32^{\circ}$ & $33^{\circ}$ \\
\hline SN-MP & $38^{\circ}$ & $39^{\circ}$ \\
\hline Gonial Angle & $135^{\circ}$ & $135^{\circ}$ \\
\hline Mx1 to NA & $4.8 \mathrm{~mm}$ & $3 \mathrm{~mm}$ \\
\hline Mx1 to NA & $26^{\circ}$ & $22^{\circ}$ \\
\hline Md1 to NB & $8 \mathrm{~mm}$ & $4 \mathrm{~mm}$ \\
\hline Md1 to NB & $27^{\circ}$ & $24^{\circ}$ \\
\hline IMPA & $91^{\circ}$ & $87^{\circ}$ \\
\hline Mx1-NF & $32 \mathrm{~mm}$ & $26 \mathrm{~mm}$ \\
\hline E-line (upper lip) & $4 \mathrm{~mm}$ & $0 \mathrm{~mm}$ \\
\hline E-line (lower lip) & $4 \mathrm{~mm}$ & $2 \mathrm{~mm}$ \\
\hline
\end{tabular}

Figure 6: Cephalometric table.

\section{Discussion}

Correction of a deep overbite is considered to be one of the most essential goals of orthodontic treatment. In patients who present with an increased vertical maxillary anterior height, maxillary incisor intrusion is currently believed to be the most trending treatment modality. Our patient had a class II malocclusion associated with deep bite owing to the vertical maxillary excess hence we intended to achieve the correction of deep bite by true intrusion of the upper anterior segment. Also the patient had a hyperdivergent growth pattern, precautions were taken to prevent the extrusion of molars and no relative mechanics were resorted to, for the intrusion of upper anterior segment. In 1989 Melsen, et al. advocated the segmented arch technique as the treatment of choice for patients with increased anterior vertical dimension. Our patient was treated with a segmented arch wire ligated only to the maxillary incisors; thereby preventing any extrusion of posterior teeth.

Conventional methods that are employed for deep bite correction are as follows:

a) Anterior Bite plate

b) Reverse curve of spee wire

c) Rickett's utility arch, designed by Robert M. Ricketts in the early 1950's which has been popularized as an integral part of bioprogressive therapy. Consists of a continuous wire that extends across both buccal segments and $t$ engages only the 1 st permanent molars and four incisors.

d) Burstone's intrusion spring, described by Burstone in 1977, consists of three parts: the posterior anchorage unit, the anterior segment and the intrusion arch.

e) K-SIR appliance by Varun Kalra, is a modification of the segmented loop mechanics. It comprises of 019"x.025" TMA archwire with closed U- loops $7 \mathrm{~mm}$ long and $2 \mathrm{~mm}$ wide. Right angle bends are placed in arch wire at level of U-loops. It is used for both intrusion and retraction.

f) Connecticut intrusion arch which is fabricated from a nickel titanium alloy. It incorporates the characteristics of utility arch as well as those of the conventional intrusion arch.

g) PG retraction spring, given by Poul Gjessing, are refabricated, standardized springs of $0.017 \times 0.025^{\prime \prime}$ or 0.016 X 0.022" wire which are used for canine retraction incisor intrusion \& retraction.

h) Cetlin's appliance.

However the mini-implant assisted intrusion with a segmented arch wire has some advantages over other conventional segmented mechanics ; first, it does not cause extrusion of the maxillary molars; this otherwise might open the mandibular plane, rotate the mandible clockwise, move mention downward and backward, and worsen a retrusive profile. Second, the patient's cooperation with wearing high-pull headgear is not required to obtain intrusion and labioversion of the incisors $[8,9]$.

Root resorption is one of the most common side effect of intrusive orthodontic movement. However, if the forces are kept light, root morphology can be preserved. In our patient, very light forces in the range of 50-60gm were used and at the end of treatment $0.5-1 \mathrm{~mm}$ of shortening of the roots of the incisors was noticed on the radiograph, which is believed to be in the acceptable range while attempting intrusion of maxillary incisors [1]. 


\section{Conclusion}

Currently, mini implant anchorage system has revolutionized orthodontics by making them perfectly stable and providing results beyond the realms of conventional orthodontic treatment. A timely follow up of the patient was done and no relapse was seen and the corrections done with the orthodontic treatment were stable. Thereby, an accurate diagnosis and treatment planning is required for the attainment of successful treatment outcome irrespective of the mechanics being executed. Segmented arch technique along with mini implant assisted incisor intrusion proves to be a promising mechanics for correction of vertical discrepancies.

\section{References}

1. Nanda R (1997) Correction of deep overbite in adults. Dent Clin North Am 41(1): 67-87.

2. Burstone CR (1977) Deep overbite correction by intrusion. Am J Orthod 72(1): 1-22.

3. Otto RL, Anholm JM, Engel GA (1980) A comparative analysis of intrusion of incisor teeth achieved in adults and children according to facial type. Am J Orthod 77(4): 437-446.
4. Hans MG, Kishiyama C, Parker SH, Wolf GR, Noachtar $R$ (1994) Cephalometric evaluation of two treatment strategies for deep overbite correction. Angle Orthod 64(4): 265-274.

5. Lindauer SJ, Lewis SM, Shroff B (2005) Overbite correction and smile aesthetics. Semin Orthod 11(2): 62-66.

6. Carillo R, Buschang PH, Opperman LA, Franco PF, Rossouw EP (2007) Segmental intrusion with miniscrew implant anchorage: a radiographic evaluation. Am J Orthod Dentofac Orthop 132(5): 576.

7. Davidovitch M, Rebellato J (1995) Two-couple orthodontic appliance systems utility arches: a twocouple intrusion arch. Semin Orthod 1(1): 25-30.

8. Ricketts RM, Bench RW, Gugino CF, Hilgers JJ, Schulhof RJ (1979) Bioprogressive therapy. Denver: Rocky Mountain Orthodontics pp: 111-126.

9. Kim TW, Kim H, Lee SJ (2006) Correction of deep overbite and gummy smile by using a mini-implant with a segmented wire in a growing Class II Division 2 patient. Am J Orthod Dentofacial Orthop 130(5): 676685. 